\title{
Natural and mixed convection in inclined channels with partial openings
}

\author{
A. Andreozzi ${ }^{1}$, B. Buonomo ${ }^{2}$, O. Manca $^{2}$ \& S. Nardini ${ }^{2}$ \\ ${ }^{I}$ DETEC - Università degli Studi di Napoli Federico II, Italy \\ ${ }^{2}$ DIAM - Seconda Università degli Studi di Napoli, Aversa (CE), Italy
}

\begin{abstract}
Natural and mixed convection in air in an inclined channel with the lower wall heated at uniform heat flux and in the presence of an obstacle either at the entrance or exit of the channel is investigated experimentally and numerically, respectively. The study is accomplished for several heat fluxes. In mixed convection the Reynolds number is equal to 150 , this being in the laminar regime. Natural convection investigation is carried out with Rayleigh number equal to $\mathrm{Ra}=6.68 \times 10^{5}$ and $2.67 \times 10^{6}$. The effect of the obstacle disposition and height is investigated by flow visualization and wall temperature distribution.

Keywords: natural convection, mixed convection, numerical and experimental analysis, inclined channels.
\end{abstract}

\section{Introduction}

Natural and mixed convection heat transfer within horizontal or inclined channels and open ended cavities has been studied widely in engineering and science applications. This is due to its importance in many applications, such as electronic cooling, fire research, solar collector systems, chemical vapor deposition systems (CVD) and nuclear reactors [1-5].

For inclined and horizontal channels heated from below, the buoyancy force can induce secondary flow that enhances the local heat transfer. Moreover, the onset point of the secondary flows is important because it delineates the region after which the two-dimensional laminar flow becomes three-dimensional and a transition motion from laminar to turbulent flow is observed [6-15]. In fact, the fluid layers heated from below can involve thermal instabilities due to the top heavy situation occurring. The buoyancy forces that overcome the stabilizing 
effects of viscous and thermal diffusion cause the onset of secondary motions. The heating of a fluid from the bottom plate in any case involves the development of secondary motions in span-wise sections. For these configurations the secondary motions imply that the flow is three-dimensional and different patterns of secondary motions can be detected for different aspect ratios and inclination angles of the plates with respect to the gravity vector.

Understanding, manipulating and controlling the secondary motions in open-ended cavities are important. There is a need for further numerical and experimental investigations on three-dimensional natural and mixed convections in cavities and particularly in horizontal and inclined channels. A number of studies investigated the instability in natural convection over inclined or slightly inclined plates heated at uniform wall temperature or heat flux as reviewed in [6-8]. Both natural and mixed convections between parallel plates have been thoroughly investigated recently, as shown in [9-11, 14-16] for mixed convection and in $[12,13,17]$ for natural convection.

The possible flow stabilization or a reduction of thermal instabilities have been investigated mainly in mixed convection [11, 18-20]. An experimental investigation on the mixed convection of air in a channel with the bottom heated horizontal wall was carried out in [18] to study the possible stabilization and elimination of the buoyancy driven unstable longitudinal, transverse and mixed vortex flow by tapering its top wall. An investigation on the mixed convection of air in a bottom heated horizontal flat duct by top plate heating experimentally was accomplished in [11] to study the possible stabilization and elimination of the buoyancy driven unstable longitudinal, transverse and mixed vortex flow. Experiments for the onset and development of the buoyancy driven secondary air flow and enhancement of heat transfer in a horizontal convergent and a divergent channel were carried out in [19]. An experimental flow visualization combined with transient temperature measurement to explore the possible stabilization of the buoyancy drive vortex flow in mixed convection in air in a bottom heated horizontal flat duct by placing a rectangular solid block on the duct bottom was carried out in [20].

It seems that the control of secondary motion in natural and mixed convection in horizontal or inclined parallel plate channels by partial opening at the inlet or outlet sections has not been investigated in depth. In this paper an experimental investigation is conducted on natural convection in inclined channels and a numerical study is carried out on mixed convection in horizontal channels, both heated from below with the inlet or outlet section partially opened.

\section{Experimental apparatus}

The experiments were carried out in natural convection. The investigated configuration was a channel with the bottom wall heated at uniform heat flux and unheated top and side walls. The heated wall consisted of a 400x530 mm sandwiched phenolic fiberboard plates. The top and side walls were made of Plexiglas. The channel spacing $b$ was measured to an accuracy of $\pm 0.25 \mathrm{~mm}$ by a dial-gauge equipped caliper. An obstruction, which was made of a glass plate 2.0 
mm thick, was located on the lower wall either at the inlet or at the outlet section as shown in Fig.1. The cavity was $400 \mathrm{~mm}$ long, $475 \mathrm{~mm}$ wide and $40.0 \mathrm{~mm}$ high and was open to the ambient along its right and left edges. In order to reduce conductive heat losses, a $150 \mathrm{~mm}$ Polystyrene block was affixed to the rear face of the heated wall. The plate facing the channel was $3.2 \mathrm{~mm}$ thick and its surface adjacent to the internal air was coated with a $35 \mu \mathrm{m}$ thick nickel plated copper layer. The low emissivity of nickel (0.05) minimized the radiation effects on heat transfer. The rear plate was $1.6 \mathrm{~mm}$ thick. Its back surface was coated with a $17.5 \mu \mathrm{m}$ thick copper layer, which was the heater. The plate was heated by passing a direct electrical current through the heater, which had a serpentine shape. Its runs were $19.6 \mathrm{~mm}$ wide with a gap of nearly $0.5 \mathrm{~mm}$ between each one, giving each heater a total length of $9.0 \mathrm{~m}$. The expected electrical resistance was $0.50 \Omega$. The narrow gaps between the runs, together with the relatively high thickness $(4.8 \mathrm{~mm})$ of the resulting low-conductive fiberglass, were suitable to maintain a nearly uniform heat flux at the plate surface. The electrical power supplied by the heater was evaluated by measuring the voltage drop across the plates and the current passing through them. To avoid electrical contact resistances, thick copper bars soldered both to the electric supply wire and to the ends of the heater were bolted together. The dissipated heat flux per board was evaluated to an accuracy of $\pm 2 \%$. The entire apparatus was located within a room, sealed to eliminate extraneous air currents.

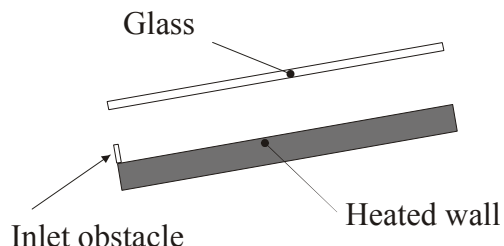

(a)

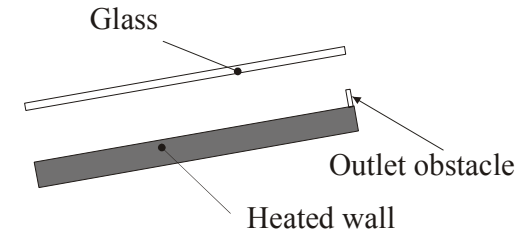

(b)

Figure 1: Investigated configurations in natural convection: (a) inlet obstacle; (b) outlet obstacle.

The wall temperatures were measured by $360.50 \mathrm{~mm}$ OD ungrounded iron-constantan thermocouples embedded in each fiberboard plate and in contact with the outer layer. They were located at twelve longitudinal stations at three different $z$ values. Fifteen thermocouples were affixed to the rear surface of the plates and embedded in the Polystyrene to enable the evaluation of conductive heat losses.

The ambient air temperature was measured by a shielded thermocouple placed near the leading edge of the channel. A Kaye instrument K170 ice point was used as a reference for thermocouple junctions. A National Instruments SCXI module data acquisition system and a PC were used for the data collection and reduction. The data acquisition was 1 b) ned through the Labview ${ }^{\mathrm{TM}}$ software. Calibration of the temperature mea .......; system showed an estimated precision of the thermocouple-readout system of $\pm 0.1^{\circ} \mathrm{C}$. 

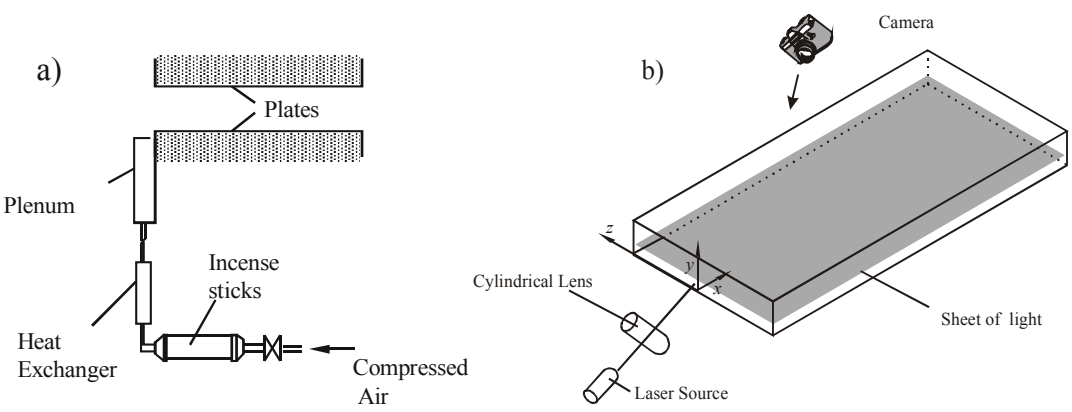

Figure 2: a) Sketch of the smoke generation arrangement; b) sketch of the visualization arrangement.

Smoke for flow visualization was generated by burning incense sticks in a steel tube, connected to a compressor. The smoke was injected through a glass heat exchanger to reduce the temperature of the smoke, and then sent into a plenum. Its temperature, measured by a thermocouple, turned out to be close to that of the ambient air entering the cavity. It was, then, driven to the test section through a small slot situated under the leading edge of the bottom plate along the plate width. A sketch of the apparatus is reported in Fig.2. The longitudinal view of the arrangement for smoke generation is reported in Fig.2a, whereas in Fig.2b the sketch of the visualization set-up is shown. Preliminary tests were carried out to determine the plenum location so as not to interfere with the air flow at the inlet section. The visualization was made possible by means of a laser sheet, generated by a He-Ne laser source. The laser sheet was produced by placing a mirror near the end of the test section at an angle of $45^{\circ}$ to the direction of the main flow, behind which a cylindrical lens was placed to enlarge the beam as needed. Small adjustments were allowed by means of a micrometer screw system, in order to take photographs at different locations along the $\mathrm{z}$ axis. The same arrangement was used to obtain pictures in the yz plane at several $x$ locations. The still camera was a digital Nikon D100 camera.

\section{Geometrical description and numerical procedure}

Numerical investigation was carried out on mixed convection in a horizontal channel. The domain was made of a principal channel and two channels with adiabatic walls, one upstream the principal channel and the other downstream, Fig.3. The principal channel was made up of a uniformly heated horizontal wall at uniform heat flux, a parallel adiabatic wall located above and two adiabatic vertical side walls. In this problem the geometrical parameters were the distance between the horizontal walls, $b$, the length of the heated plate, $L$, the width of the channel, $\mathrm{W}$, and the height of the obstacle, $\mathrm{h}$. The characteristic dimensionless numbers were the Reynolds number, the Rayleigh number and the Richardson number and they are based on the distance $\mathrm{b}$ between the horizontal plates. The 
aim of this paper was to investigate the effect of Reynolds and Rayleigh numbers on thermal and fluid dynamic behavior of mixed convection in a horizontal channel heated from below and the effect of the obstacle in the inlet or outlet section. The mixed convective flow in the horizontal channel was considered to be incompressible. The analysis was transient and the thermophysical properties were assumed constant with temperature except for the density, as suggested by the Boussinesq approximation. The thermophysical properties of the fluid were evaluated at the ambient temperature, $\mathrm{T}_{0}$, which was equal to $300 \mathrm{~K}$.

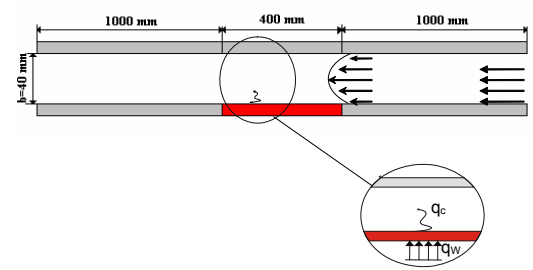

Figure 3: Configuration numerically investigated.

The commercial CFD code Fluent 6.2 [21] was employed to solve the governing equations. The imposed boundary conditions were: uniform heat flux and no-slip condition on the lower plate; adiabatic wall and no-slip condition on the other solid walls; pressure inlet or pressure outlet conditions on the openboundaries. Computations were made for $\mathrm{q}_{\mathrm{w}}=150 \mathrm{~W} / \mathrm{m}^{2}, \mathrm{~L}=400 \mathrm{~mm}, \mathrm{~b}=40.0$ $\mathrm{mm}$, and $\mathrm{W}=500 \mathrm{~mm}$. The employed grid was uniform inside the horizontal channel consisting of $51 \times 21 \times 41$ points, whereas they decreased with an exponential law inside the auxiliary channels. This grid was the right compromise between the solution accuracy and the computational time. About the time step a value of $10^{-1}$ was employed after some tests on different time steps. This analysis was accomplished for the highest considered Rayleigh number in order to obtain a valid test also for lower Ra values.

\section{Results and discussion}

Experimental investigations were carried out for $\mathrm{q}_{\mathrm{w}}=60$ and $240 \mathrm{~W} / \mathrm{m}^{2}, \theta=10^{\circ}$, $\mathrm{b}=40.0 \mathrm{~mm}$ and $\mathrm{h}=10.0$ and $20.0 \mathrm{~mm}(\mathrm{~h} / \mathrm{b}=0.25$ and 0.50$)$.

In Fig. 4 visualization of flow motion along the longitudinal section with and without obstacle is reported for $\mathrm{q}_{\mathrm{w}}=60$ and $240 \mathrm{~W} / \mathrm{m}^{2} \quad\left(\mathrm{Ra}=6.68 \times 10^{5}\right.$ and $\left.2.67 \times 10^{6}\right)$. When the obstacle is absent, $\mathrm{h} / \mathrm{b}=0$, fluid moves adjacent the lower wall up to about $1 / 3 \mathrm{~L}$ where it separates and moves toward the upper wall. Then it advances close the unheated upper wall. For the lowest value of the Rayleigh number separation occurs closer to the exit section than for the highest Rayleigh number value. When the inlet obstacle, is present a recirculation zone develops and fluid joins again the lower wall at distance from the inlet section equal to about $\mathrm{b}$ for $\mathrm{h} / \mathrm{b}=0.25$, whereas for $\mathrm{h} / \mathrm{b}=0.5$ this distance is much higher. When the obstacle is at exit section separation happens similarly to the case with no 
obstacle, even if for $\mathrm{Ra}=2.67 \times 10^{6}$ the $\mathrm{x}$ value, where separation takes place, is higher. However, the obstacle presence slows down the flow. For the highest Rayleigh number value separation seems to take place more distant the inlet section.
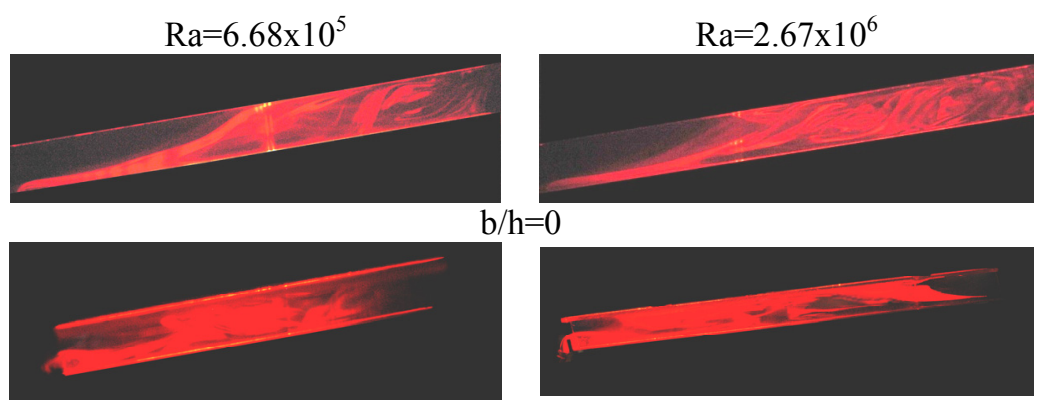

$\mathrm{b} / \mathrm{h}=0.25$, inlet obstacle
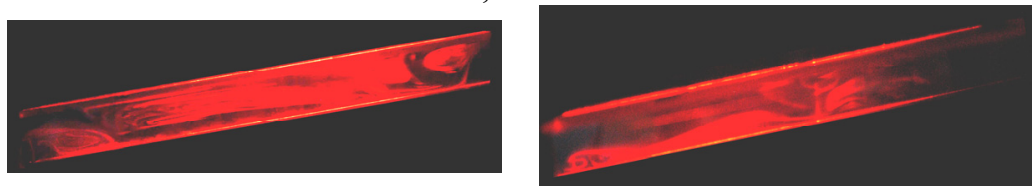

$\mathrm{b} / \mathrm{h}=0.50$, inlet obstacle

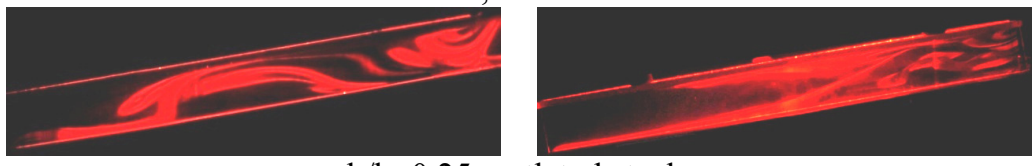

$\mathrm{b} / \mathrm{h}=0.25$, outlet obstacle
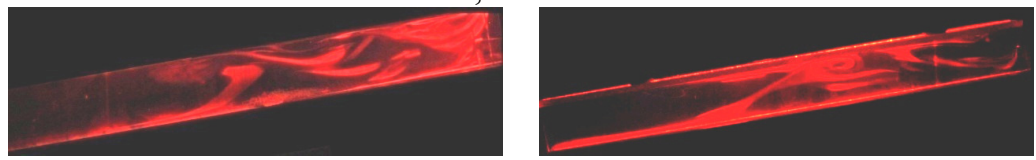

$\mathrm{b} / \mathrm{h}=0.50$, outlet obstacle

Figure 4: Flow visualization in longitudinal section $\mathrm{z}=0$ for two Rayleigh numbers.

Separation from lower wall determines secondary motions which develop along the channel. They are very clear from flow visualization in channel transversal sections. In Fig.5 pictures of flow visualization in configurations without obstacle and with obstacle at outlet section and $\mathrm{h} / \mathrm{b}=0.5$ are reported. The considered sections are at 100, 200 and $395 \mathrm{~mm}$ from the inlet channel section. In the section at $\mathrm{x}=100 \mathrm{~mm}$ and $\mathrm{Ra}=6.68 \times 10^{5}$, secondary motions start to develop. This is clearer when the obstacle is absent. For the highest value of Ra plumes are present at $\mathrm{x}=100 \mathrm{~mm}$ which extend for no more $1 / 3 \mathrm{~b}$ from the lower wall. When the obstacle is present secondary motions are not present at $x=100$ $\mathrm{mm}$. At $\mathrm{x}=200$ and $395 \mathrm{~mm}$ secondary motions occur in each considered configuration. They are less developed and intense when the outlet obstacle is present. 


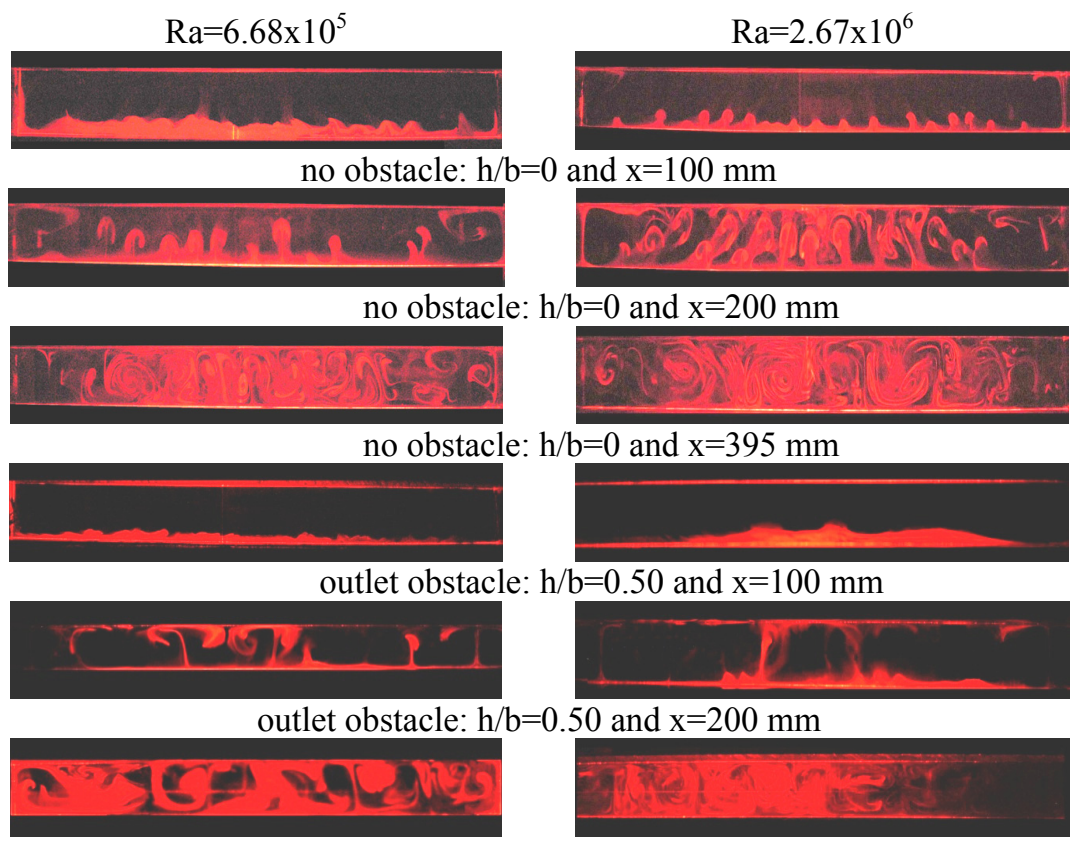

outlet obstacle: $\mathrm{h} / \mathrm{b}=0.50$ and $\mathrm{x}=395 \mathrm{~mm}$

Figure 5: Flow visualization in several transversal sections and for two Rayleigh numbers.

Results of numerical investigation on mixed convection in horizontal channel are reported in Figs. 6-8 for $\mathrm{Re}=150$ and $\mathrm{Ra}=1.67 \times 10^{6}$ when an inlet or outlet obstacle with $\mathrm{h} / \mathrm{b}=0.5$ is present or not. Velocity vectors in the longitudinal section $\mathrm{z}=0 \mathrm{~mm}$, in Fig.6, show the differences among the considered configurations. Particularly, for the no obstacle case, in Fig.6a, flow separates from the lower wall at about $\mathrm{x}=250 \mathrm{~mm}$ and, at this location, a recirculation zone is evident close to the upper wall. When an obstacle is present at the inlet section, in Fig.6b, a vortex behind it is noticed and at about a distance b from inlet channel section flow joins again the lower wall. In this configuration flow does not separate from the lower wall. When an obstacle is located at the exit, in Fig.6c, flow separates from the lower wall at about $\mathrm{x}=340 \mathrm{~mm}$ and, hence, close to the exit.

Temperature fields in transversal sections at $\mathrm{x}=0,100,200,300$ and $400 \mathrm{~mm}$ are presented in Figs.7a-7c. When there is not an obstacle, in Fig.7a, secondary motions are developing in the section at $x=100 \mathrm{~mm}$ close to the side walls whereas at $x=200 \mathrm{~mm}$ in the whole section secondary effects are clear. At $x=300$ $\mathrm{mm}$ secondary motions are present in the entire section and at $\mathrm{x}=400 \mathrm{~mm}$ temperature is quasi-uniform in the section. When an obstacle is present at the inlet section, in Fig. $7 \mathrm{~b}$, at $\mathrm{x}=100 \mathrm{~mm}$ plumes are developing whereas secondary motions are absent at side zones. Temperature close to the heated wall seems to 
be less uniform than in the configuration without obstacle. At higher $\mathrm{x}$ values plumes appear more developed. In the configuration with an obstacle at the channel exit, in Fig.7c, channel behavior, in terms of air temperature and secondary motions, seems very similar to that for the channel without obstacle, reported in Fig.7a. In fact, at the channel inlet section temperature increases are not evident and at $\mathrm{x}=100 \mathrm{~mm}$ air temperature value increase only in the central zone of the transversal section whereas secondary motion develops in the zone adjacent the side walls.

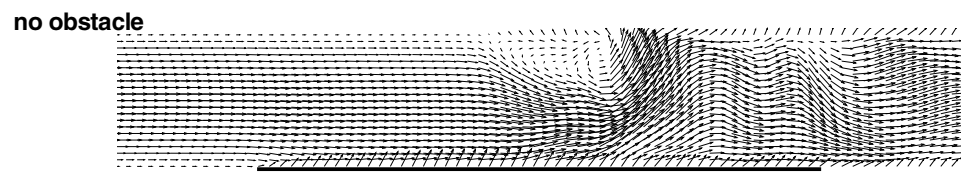

(a)

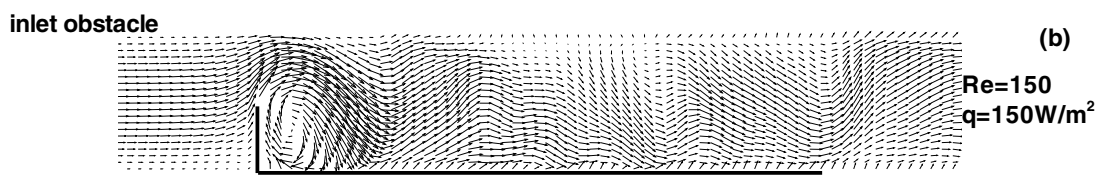

outlet obstacle

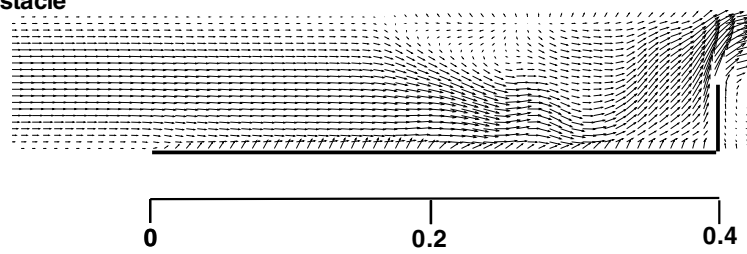

(c)

Figure 6: Velocity vectors in the longitudinal plane $\mathrm{z}=0 \mathrm{~mm}$ for $\mathrm{Re}=150$ and $\mathrm{q}_{\mathrm{w}}=150 \mathrm{~W} / \mathrm{m}^{2}$ : (a) no obstacle; (b) inlet obstacle; (c) outlet obstacle.

Finally, in Fig.8 surface temperatures on the heated wall is shown for the configurations with obstacle. The great difference between the two cases is clear. In fact, when the obstacle is located at the inlet section, in Fig.8a, warmer zones are present close to the entrance whereas when the obstacle is at the outlet section higher temperature values take place in the zone between $\mathrm{x}=100 \mathrm{~mm}$ and $200 \mathrm{~mm}$.

\section{Conclusions}

An experimental investigation on natural convection in air, in an inclined channel, with the lower wall heated at uniform heat flux and an obstacle located either at inlet or outlet section of the channel was carried out. Results of flow visualization highlighted the effect of secondary motions along the heated wall 


\section{$\mathrm{Re}=\mathbf{1 5 0}$ \\ $q=150 \mathrm{~W} / \mathrm{m}^{2}$ \\ no obstacle}

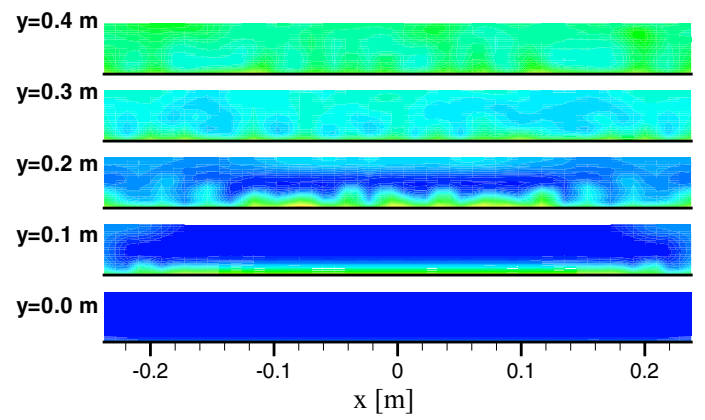

(a)

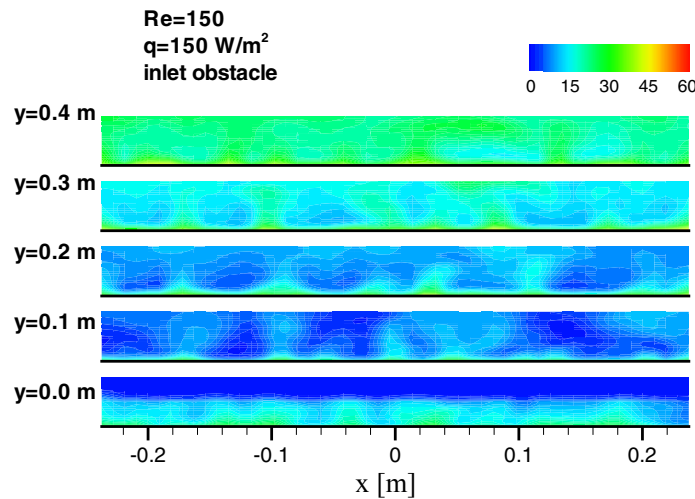

(b)

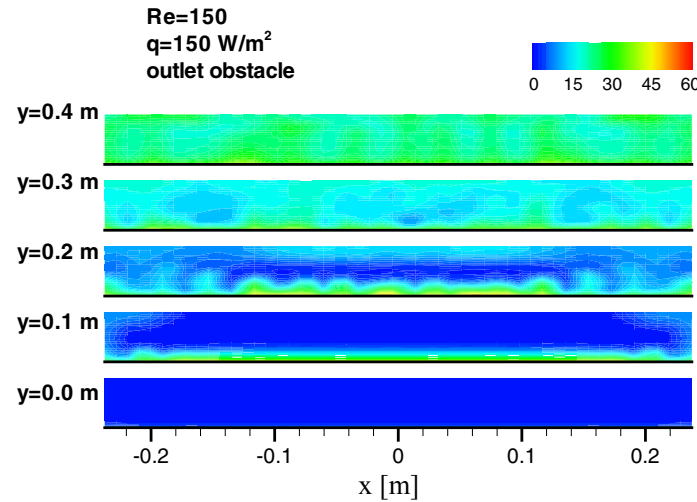

(c)

Figure 7: Temperature fields in transversal sections for $\mathrm{Re}=150$ and $\mathrm{q}_{\mathrm{w}}=150$ $\mathrm{W} / \mathrm{m}^{2}$ : (a) no obstacle; (b) inlet obstacle; (c) outlet obstacle. 


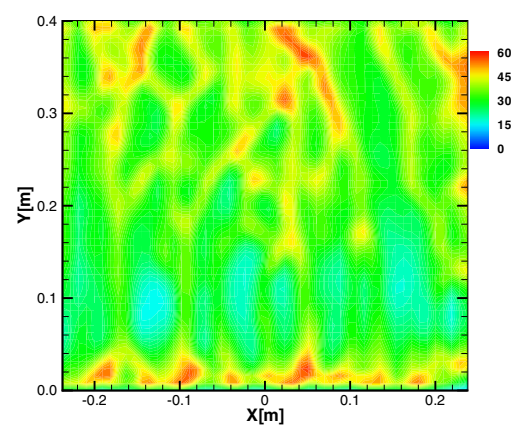

(a)

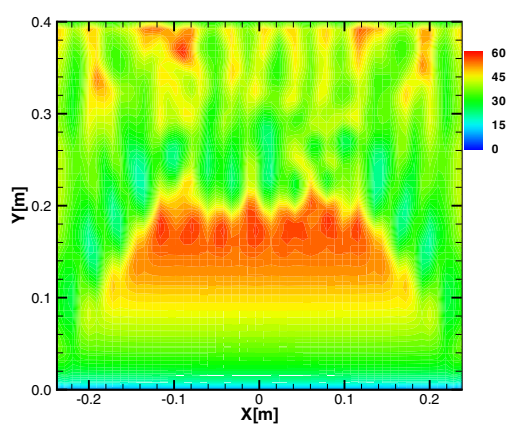

(b)

Figure 8: Temperature field on the heated wall for $\mathrm{Re}=150$ and $\mathrm{q}_{\mathrm{w}}=150$ $\mathrm{W} / \mathrm{m}^{2}$ : (a) inlet obstacle; (b) outlet obstacle.

and the separation from the lower heated plate strongly depended on the location and height of the obstacle. When the obstacle is at the channel entrance a recirculation zone develops behind it. When the obstacle is at the exit section, flow motion is similar to the one in case with no obstacle

Furthermore, a numerical investigation was accomplished on mixed convection in horizontal channel with or without obstacle. For the no obstacle case a recirculation zone was present in the central part of the channel whereas for the case of the obstacle at the entrance the recirculation was behind it. When the obstacle was at the exit no recirculation was detected.

\section{Acknowledgement}

This work is funded by the Regione Campania Legge 5 and SUN 2007 research grants.

\section{Nomenclature}

b channel spacing, $\mathrm{m}$

g acceleration due to the gravity, $\mathrm{ms}^{-2}$

Gr Grashof number,

$$
=\frac{g \beta q_{c} b^{4}}{v^{2} k}
$$

$\mathrm{h}$ height of the obstacle, $\mathrm{m}$

$\mathrm{k}$ thermal conductivity, $\mathrm{Wm}^{-1} \mathrm{~K}^{-1}$
L length of the heated wall, $\mathrm{m}$

Pr Prandtl number

q heat flux, $\mathrm{Wm}^{-2}$

Ra Rayleigh number, $=\mathrm{Gr} P \mathrm{Pr}$

Re Reynolds number, $=\frac{u_{i} b}{v}$

T temperature, $\mathrm{K}$ 
$\mathrm{u}_{\mathrm{i}} \quad$ average velocity at inlet section of the channel, $\mathrm{ms}^{-1}$

x horizontal coordinate distance, $\mathrm{m}$

y vertical coordinate distance, $\mathrm{m}$

z coordinate along the width of the plates, $\mathrm{m}$

W width of the plate, $\mathrm{m}$
Greek symbols

$\beta$

volumetric coefficient of expansion, $\mathrm{K}^{-1}$

$\theta$ inclination angle from horizontal, ${ }^{\circ}$

$v \quad$ kinematic viscosity, $\mathrm{m}^{2} \mathrm{~s}^{-1}$

Subscript

c convective

w wall

\section{References}

[1] Gebhart, B., Jaluria, Y., Mahajan, R. \& Sammakia, B., Buoyancy-Induced Flows and Transport, Hemisphere Publishing Corporation, Washington, D.C, 1988.

[2] Manca, O., Morrone, B., Nardini, S. \& Naso, V., Natural convection in open channels, in Computational analysis of convection heat transfer, WIT Press, Southampton, Boston, 2000.

[3] Bianco, N., Morrone, B., Nardini, S. \& Naso, V., Air natural convection between inclined parallel plates with uniform heat flux at the walls, Journal of Heat and Technology, 18 (2), pp. 23-45, 2000.

[4] Kasayapanand, N. \& Kiatsiriroat, T., Enhanced heat transfer in partially open square cavities with thin fin by using electric field, Energy Conversion and Management, 50 (2), pp. 287-296, 2009.

[5] Chong, D., Liu, J. \& Yan, J., Effects of duct inclination angle on thermal entrance region of laminar and transition mixed convection, International Journal of Heat and Mass Transfer, 51, pp. 3953-3962, 2008.

[6] Jeschke, P., \& Beer, H., Longitudinal vortices in a laminar natural convection boundary layer flow on an inclined flat plate and their influence on heat transfer, Journal of Fluid Mechanics, 432, pp. 313-339, 2001.

[7] Kimura, F., Yoshioka, T., Kitamura, K., Yamaguchi, M. \& Asami, T., Fluid flow and heat transfer of natural convection at a slightly inclined, upwardfacing, heated plate, Heat Transfer-Asian Research, 31, pp. 362-375, 2002.

[8] Biertümpfel, R. \& Beer, H., Natural convection heat transfer increase at laminar-turbulent transition in a presence of instationary longitudinal vortices, International Journal of Heat and Mass Transfer, 46, pp. 3109$3117,2003$.

[9] Nicolas, X., Revue bibliographique sur les écoulements de PoiseuilleRayleigh-Bénard: écoulements de convection mixte en conduites rectangulaires horizontales chauffées par le bas, International Journal of Thermal Sciences, 41, pp. 961-1016, 2002.

[10] Lin, T. F., Buoyancy driven flow and thermal structure in a very low Reynolds number mixed convective gas flow through a horizontal channel, International Journal of Heat and Fluid Flow, 24, pp. 299-309, 2003. 
[11] Chen, S. W., Chang, C. Y., Lir, J. T. \& Lin, T. F., Stabilization and elimination of transient unstable mixed convective vortex flow of air in a bottom heated horizontal flat duct by top plate heating, International Journal of Heat and Mass Transfer, 47, pp. 4137-4152, 2004.

[12] Andreozzi, A., Buonomo, B., Manca, O. \& Nardini S., Three dimensional transient natural convection in horizontal channels heated from below, Proc. Energy: production, distribution and conservation, 2, pp. 773-782, Milan - May 14-17, 2006.

[13] Andreozzi, A., Buonomo, B., Manca, O. \& Nardini, S., Experimental investigation on the effect of longitudinal aspect ratio on natural convection in inclined channels heated below, Proc. $8^{T H}$ Biennial Conference on Engineering Systems Design and Analysis, paper ESDA2006-95526, Torino, Italy, July 19-22, 2006.

[14] Tuh, J. L., Transverse recirculations in low Reynolds number mixed convective gas flow over a model heated substrate, Experimental Thermal and Fluid Science, 32, 293-308, 2007.

[15] Chiu, H. C. \& Yan, W. M., Mixed convection heat transfer in inclined rectangular ducts with radiation effects, International Journal of Heat and Mass Transfer, 51, pp. 1085-1094, 2008.

[16] Foglia G., Lazzari S. \& Manca O, Experimental investigation on mixed convection in a horizontal channel, Journal of Heat and Mass Transfer, 1, pp. 27-48, 2007.

[17] Andreozzi, A., Buonomo, B., Manca, O. \& Nardini, S., Effect of transversal aspect ratio on natural convection in an inclined channel with heated bottom wall, Proc. of $13^{\text {th }}$ Int. Flow Visualization Symposium, paper n. 193080424, July 1st- 4th, Nice French Riviera, 2008.

[18] Tseng, W. S., Lin, W. L., Yin, C. P., Lin, C. L. \& Lin, T. F., Stabilization of buoyancy-driven unstable vortex flow in mixed convection of air in a rectangular duct by tapering its top plate, Journal of Heat Transfer, 122 , pp.58-65, 2000.

[19] Liu, C. W. \& Gau, C., Onset of secondary flow and enhancement of heat transfer in horizontal convergent and divergent channels heated from below, International Journal of Heat and Mass Transfer, 47, pp. 54275438, 2004.

[20] Chen, S.W., Shu, D.S., Lir, J.T. \& Lin, T.F., Buoyancy driven vortex flow and its stability in mixed convection of air through a blocked horizontal flat duct heated from below, International Journal of Heat and Mass Transfer, 49, pp. 3655-3669, 2006.

[21] Fluent Incorporated, Fluent 6.2, User Manual, 2006. 